\title{
MEASUREMENT OF SENSITIVITY OF DIFFERENT WAVE MODES TO SUBSURFACE DEFECTS
}

\author{
Abdullah Atalar $\dagger$, Hayrettin Köymen, and Oğuz Yemiş̧̧iler \\ Middle East Technical University, Ankara, TURKEY \\ † Bilkent University, Ankara TURKEY
}

\begin{abstract}
Excitation of acoustic wave modes in a layered solid is investigated when the solid is immersed in a liquid. This is done by computing the reflection coefficient of acoustic plane waves at the liquid-layered-solid interface. Then, a method to evaluate the sensitivity of various modes supported in the layered structure to subsurface defects is introduced. The method involves insonification of the layered structure with a conical wave whose axis coincides with the defect. Since all the rays in the conical wavefront hit the planar interface at the same angle, it is possible to excite a single kind of mode in the layer. By adjusting the angle of inclination of the cone, it is possible to excite the modes selectively. Since the conical waves converge to a line focus at the cone axis, the excited mode will focus on the defect. By recording the reflected signal amplitude as the cone angle is varied, a curve is obtained from which it is possible to conclude the sensitivity of various modes to the subsurface flaw. The results of such measurements indicated that the generalized Lamb wave modes are more sensitive to subsurface defects than the Rayleigh waves. An imaging system which makes use of focused Lamb waves was built and the system produced images of very small subsurface defects.
\end{abstract}

\section{INTRODUCTION}

Structures having layers made up of different materials are frequently used in industry. Adhesion properties of layers on the substrate is of critical importance. Observation of the interface between layers through possibly opaque materials is very difficult. Use of bulk acoustic waves for the purpose of imaging flaws and discontinuities is complicated by the presence of multiple internal reflections, the occurrence of surface waves, and the transformation of longitudinal waves into shear waves. Although pulse-echo methods, in conjunction with holographic imaging, may be used to reject multiply reflected signals and thus simplify the interpretation, the obtained images are in general very difficult to interpret even for the simple shaped solids. On the other hand, use of surface acoustic waves for the imaging of such structures is limited to a region very close to the surface. Moreover, surface acoustic waves are rather insensitive to the inhomogeneities at interfaces several wavelengths below the surface. Layered structures support additional types of acoustic waves apart from bulk and surface waves. When the structure is immersed in a liquid, these modes can be excited by waves generated in the liquid, incident at a critical angle.

In this paper, (i) we investigate theoretically the excitation of various layer modes in the solid structure by the bulk waves in the immersion liquid; (ii) we introduce a method to evaluate the reflection sensitivity of various modes to subsurface defects; and (iii) a method of imaging subsurface interfaces by one of the sensitive modes.

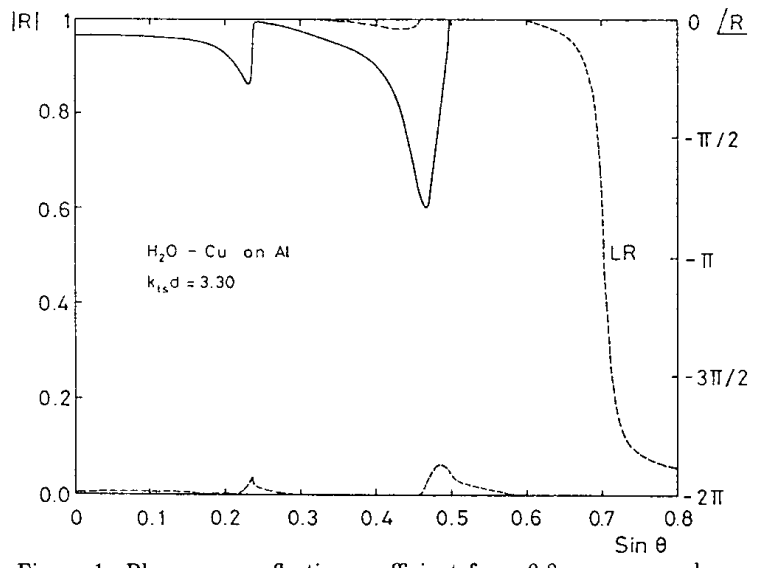

Figure 1: Plane wave reflection coefficient for a $0.8 \mathrm{~mm}$ copper layer on an aluminum substrate at $2 \mathrm{MHz}\left(k_{t s} d=3.30\right)$.

\section{EXCITATION OF LAYER MODES}

It is well known that the Rayleigh waves can be excited on the planar surface of a solid material immersed in a liquid by bulk waves incident on the surface from the liquid side, provided that the angle of incidence is at the Rayleigh critical angle [1]. This forms the basis of the wedge transducers $[2,3,4]$. The excitation of a Rayleigh wave on the surface exhibits itself as a phase transition at the critical angle in the plane wave reflection coefficient as a function of incidence angle [5].

Waves excited in a layered soiid structure will be either bulk waves or leaky interface waves. The leaky waves can be in the form of leaky Rayleigh waves or layer waves such as generalized Lamb waves [6]. The reflection coefficient at the liquid-layered-solid interface exhibits phase transitions corresponding to these modes. The angular positions of the phase transitions can be used to predict the incidence angle of the bulk wave in the liquid for efficiently exciting the respective layer modes.

An analytic expression for the reflection coefficient at the liquid-layeredsolid interface for a single layer is given in the literature $[7,8]$. We computed this reflection coefficient for some layered structures. Fig. 1 shows the reflection coefficient amplitude and phase between water and a copper layer on an aluminum substrate for $k_{t}, d=3.30$, where $k_{t s}$ is the shear wavenumber of the substrate and $d$ is the layer thickness. As seen in the figure, for a thin layer of copper there exists a single transition in the phase which corresponds to a leaky Rayleigh-like (LR) wave. For this particular case, this mode is slower than the Rayleigh wave on the free copper surface. As the layer thickness is increased (see Fig. 2), a second mode (M2) appears as indicated by the second phase transition in the reflection coefficient. This mode is a generalized 


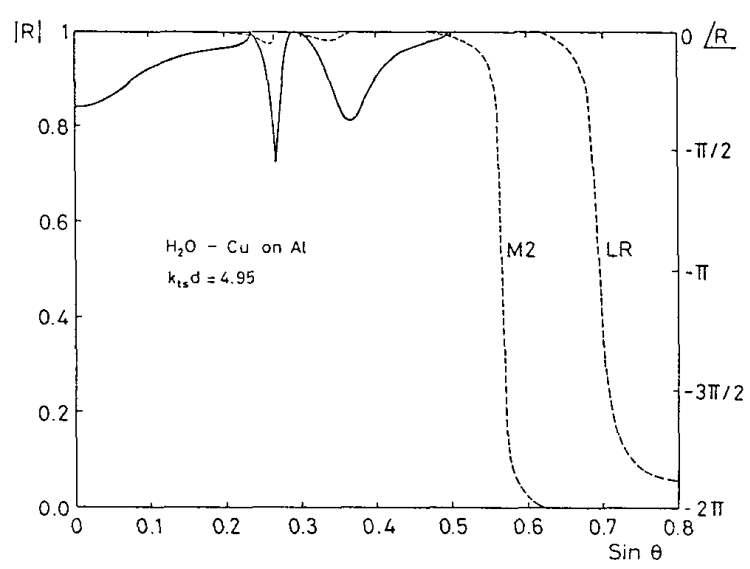

Figure 2: Plane wave reflection coefficient for a $1.2 \mathrm{~mm}$ copper layer on an aluminum substrate at $2 \mathrm{MHz}\left(k_{t} d=4.95\right)$.

Lamb wave which is leaky due to the presence of the fluid. A second generalized Lamb wave mode (M3) emerges when the layer thickness is further increased as depicted in Fig. 3. The existence of various modes and their excitation angles are compactly presented in Fig. 4 as a function of the $k_{t s} d$. This figure is actually a different way of displaying dispersion relation [9]. Note that, it depicts only the leaky waves, it does not show bulk waves which may also be excited. LR mode has a wave speed close to that of Rayleigh wave in the substrate material when the layer is very thin. As the layer thickness is increased the phase velocity decreases rapidly, and it becomes smaller than the phase velocity of the Rayleigh wave in the layer material. As the layer thickness is further increased, the wave gets faster and it approaches to the speed of the Rayleigh wave in the layer material. M2 mode exists only for layer thicknesses above a certain critical value. At the cutoff point the wave speed is equal to that of the shear wave in the substrate material, and for a thicker layer it approaches to the shear wave speed of the layer material. M3 and higher modes have a very similar behavior to M2 mode except that they exist for even thicker layer widths.

It was shown that only a single mode (LR) can exist when the layer material has a higher transverse speed than that of the substrate [10]. Hence, an aluminum layer on a copper substrate would not support generalized Lamb wave modes.

\section{SENSITIVITY MEASUREMENT OF MODES TO SUBSURFACE DEFECTS}

The defects in a multilayered structure can exist in the layer, on the interface or under the interface. Our aim is to determine the most sensitive wave mode for detection of these defects. As shown in Fig. 5, the method proposed here involves first the generation of a conical wave with the cone axis perpendicular to the layered surface under examination. The technique of obtaining conical wavefronts was described elsewhere [11]. Such a conical wave has the property that the conical nature will be preserved upon transmission through any planar interface perpendicular to the cone axis, regardless of the mode into which it is converted. This is due to the fact that all the rays in the wavefront have the same angle of incidence with respect to the planar interface. On the contrary, spherical waves will not preserve their shape upon transmission through planar boundaries. The excited conical bulk (longitudinal or shear) waves form a line focus with a linear phase in the solid ma-

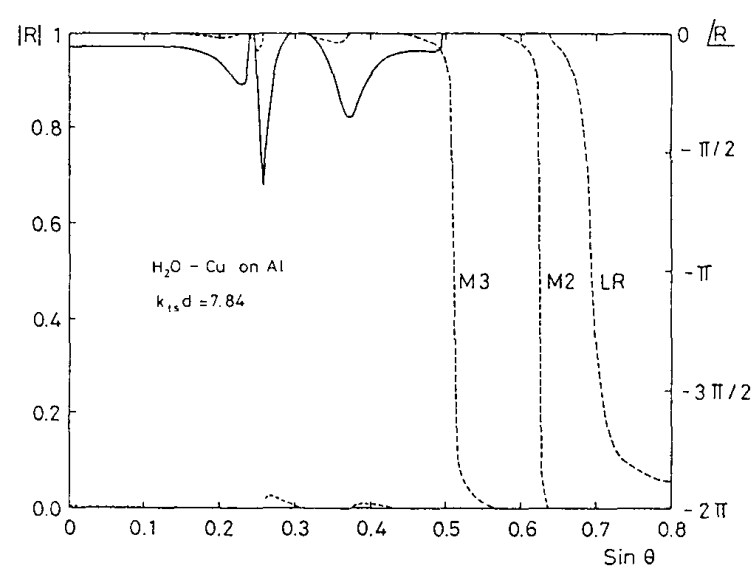

Figure 3: Plane wave reflection coefficient for a $1.9 \mathrm{~mm}$ copper layer on an aluminum substrate at $2 \mathrm{MHz}\left(k_{t} d=7.84\right)$.

terial, but for most cases they do not get excited with a high efficiency because of the impedance mismatch between the immersion liquid and the material. For some angles it may be possible to excite shear waves with a reasonable efficiency. On the other hand, leaky layer waves are excited with a high efficiency. Since the excited Lamb waves propagate parallel to the interface, they form a cylindrical wave converging to a line focus. The focal line will be normal to the surface and its height will be limited by the layer width. For minimum loss in a pulse-echo arrangement, the lateral size of the conical wave must be proportional to the Schoch displacement for the particular mode [11].

The measurement technique can be implemented by aligning the focal line of the conical wave with the subsurface defect (Fig. 5). The reflected waves from the defect leak back into the liquid medium and subsequently detected. The detected signal amplitude is recorded as the angle of conical wave is varied. When the received signal amplitude is plotted as a function of incidence angle, the resulting curve, which we call $V(\theta)$, can be used to deduce the relative sensitivities of the excited modes and the most sensitive excitation angle for detection of the particular defect.

Fig. 6 depicts measurement results for $V(\theta)$ for a copper layer on an aluminum substrate with an artificial flaw at the interface between the copper and the aluminum. The flaw is $3 \mathrm{~mm}$ diameter and $1 \mathrm{~mm}$ deep indentation in the aluminum substrate hidden by the $1.12 \mathrm{~mm}$ thick copper layer. Measurements are repeated at different frequencies which corresponds to $k_{t s} d$ values of $3.70,4.16,4.62$ and 5.08. The curves are normalized with respect to the response of the transducer at the measurement frequencies. As the $k_{t}, d$ value is increased the peak of the curves shift toward higher $\theta$ values. Comparison of the curves with Fig. 4 shows that the peaks are associated with the M2 mode (leaky generalized Lamb wave). The peak related to the Rayleigh-like wave around 44 degrees is not significant. The Schoch displacement parameter for the Lamb wave mode is considerably higher than that for the Rayleigh-like mode [10]. In the experiment, the lateral size of the conical wave was optimized for LR mode. Therefore, there would be more conversion loss for M2 mode excitation. Inspite of this fact, the M2 mode peak is much higher than the LR mode peak. This shows that there is very little reflection of Rayleigh-like wave from the defect. Notice also that the magnitude of the peak is considerably different for different $k_{t s} d$ values. This may be due to varying conversion efficiency of conical wedge at different frequencies, or due to varying reflection coefficient at the defect.

The correspondence between the $V(\theta)$ curves and Fig. 4 is good but

772 - 1988 ULTRASONICS SYMPOSIUM 


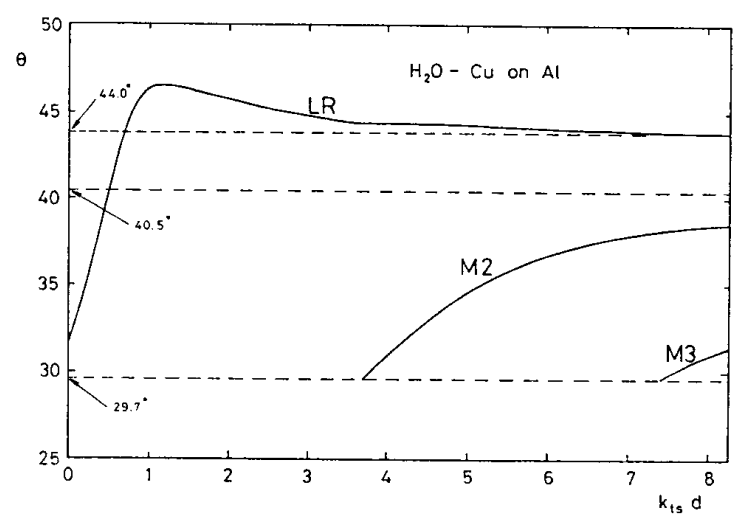

Figure 4: Excitation angle of various modes from the liquid side as a function of copper layer thickness-wavenumber product on an aluminum substrate

not perfect. The small disagreement may be a consequence of the fact that the conical wave in the experiment is not ideal. The angle that the rays of the conical wave makes with the normal to the surface has an angular variance of 4 degrees. This causes an integration effect within the angular coverage of the beam. An examination of Fig. 1 shows that in addition to leaky waves it is possible to excite shear waves with high efficiency at angles close to 30 degrees. Moreover, these waves differ in phase from the Lamb waves by 180 degrees upon reflection. Hence, if shear waves and Lamb waves are excited simultaneously, then the reflected waves will add destructively on the transducer surface. The $V(\theta)$ curves include the consequences of such interference. Supporting this fact, in some cases pulsed measurements revealed two pulses which interfered with each other visible on the oscilloscope screen. To get a better delineation of various modes in $V(\theta)$ curves, one needs a more accurate conical wave.

As the particular layer mode impinges on the defect which can be of any shape or character, a very complicated reflection process takes place [12] and a multitude of modes will be reflected. The reflected modes could be longitudinal waves, shear waves, Rayleigh waves or Lamb waves. In the proposed measurement technique, the output signal amplitude is proportional only to the amplitude of the reflected interface mode which was originally excited. The other reflected modes will not contribute anything significant to the output signal, because they will not be able to form the original conical wave in the liquid medium.

\section{IMAGING USING FOCUSED LAMB WAVES}

As seen in the previous section, generalized Lamb waves exist under certain conditions. For their presence, the shear wave speed of the layer material must be less than that of the substrate material and the layer must be thick enough. If they exist, they provide potentially higher sensitivity to defects at the interfaces compared to the Rayleigh wave. This fact can be exploited to obtain images of subsurface defects with high sensitivity.

An imaging system based on mechanical scanning [13] was built which scans the surface with focused Lamb waves. A phantom was machined to test the potential of Lamb waves to subsurface defects. Indentations - to simulate flaws - of depth $0.6 \mathrm{~mm}$, and $1.0 \mathrm{~mm}$ were milled on an aluminum surface on which a copper layer of $1.12 \mathrm{~mm}$ thickness was epoxy-bonded to hide these artificially induced flaws. This phantom

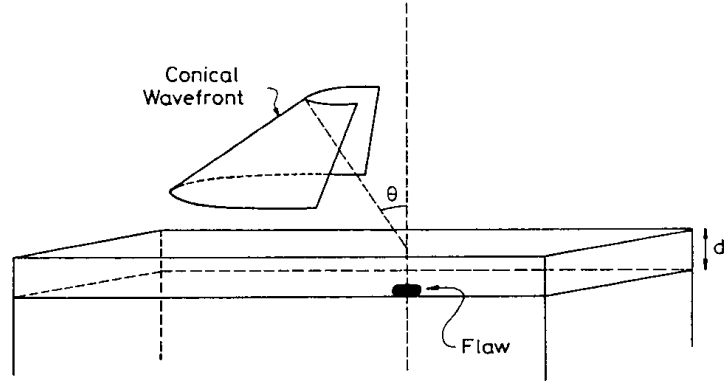

Figure 5: Geometry for $V(\theta)$ measurement

was imaged with an incidence angle of 34 degrees $(2 \mathrm{MHz})$ with a $k_{t s} d$ of 4.62 (Fig. 7). In the image higher signal levels are mapped to darker pixels. The excited mode corresponds to a generalized Lamb wave in the copper layer. This Lamb wave image clearly shows all the hidden indentations on the aluminum surface. The received signal level for $1 \mathrm{~mm}$ deep indentation is higher than that for the $0.6 \mathrm{~mm}$ deep, which is an expected result. But, the signal obtained from a $1.5 \mathrm{~mm}$ deep indentation (not seen in the image) is lower than that from the $1 \mathrm{~mm}$ deep. In addition to the indentations, there is a significant structure in the image which is probably due to the imperfections in the epoxybond. If the angle of incidence of the conical wave is adjusted around 30 degrees or lower to excite shear waves, the resulting image shows only the indentations, but not the additional structure. Therefore, the image shown in Fig. 7 is truly an interface image. It shows the inhomogeneities in the interface between the layer and substrate and in the layer itself

Experimentation with other phantoms indicated that the sensitivity of the system is very good. It was possible to detect $0.1 \mathrm{~mm}$ deep indentations below $1.6 \mathrm{~mm}$ thick copper layer with a high signal-tonoise ratio. This is a good result since the wavelength of shear waves in the substrate is about $1.5 \mathrm{~mm}$.

We must point out that the shapes of detected structures in the image are more related to the response of the imaging system than the defects themselves. The system has a good resolution in horizontal direction, but it has a poor vertical resolution. That is why the circular indentations show up as elongated ellipses in the image. The vertical resolution can also be improved by proper signal processing and making use of time of flight data [14]. The signal to noise ratio of the received signal is very good, although no optimization [11] of conical wave size has been done. It is possible to increase the signal level considerably after the optimization. Finally, we must add that the indentations in this phantom are not visible under an X-ray imaging system.

\section{CONCLUSIONS}

We have introduced a method to selectively measure the sensitivity of excited modes to small defects in a layered structure. This method results in a curve called $V(\theta)$ which has a morphology depending on the geometrical and elastic properties of the layered structure and of the defect. From this curve one can ascertain the most sensitive mode and hence the most suitable excitation angle for the particular type of defect.

$V(\theta)$ curves are affected by several factors: Conversion efficiency of bulk waves to the excited mode, reflection coefficient of the particular interface mode from the defect, the shape of the defect. $V(\theta)$ curves provide the sensitivity information in the most appropriate combination of the above factors. The maximum response point on the curves 


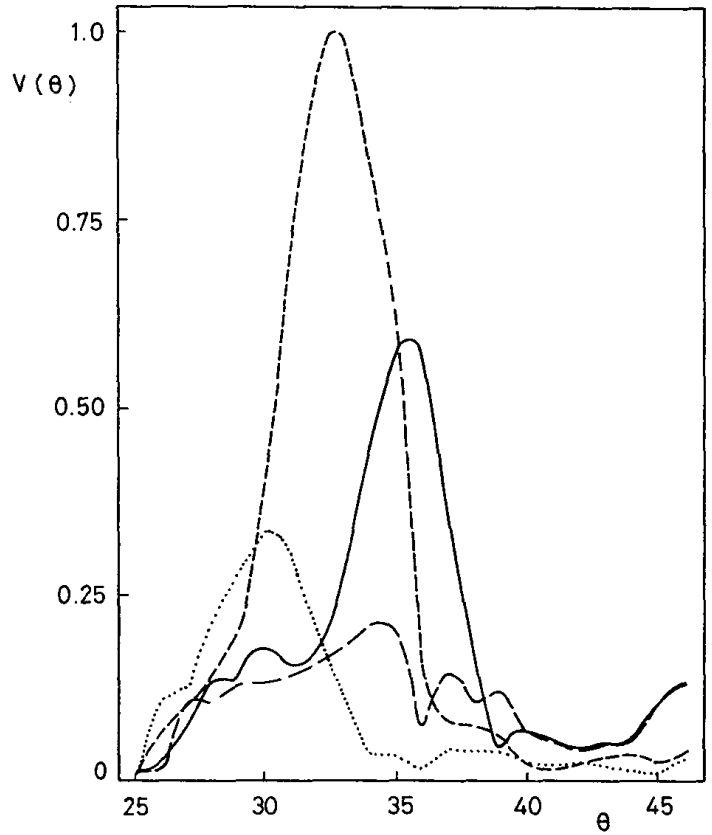

Figure 6: Measured $V(\theta)$ curves for a $1.12 \mathrm{~mm}$ thick copper layer on aluminum substrate at $k_{t s} d=3.70$ (dotted), 4.16 (dashed), 4.62 (solid) and 5.08 (long dashed). Defect is a $3 \mathrm{~mm}$ diameter and $1 \mathrm{~mm}$ deep indentation in the substrate.

will indicate the most sensitive incidence angle for waves in the liq uid medium. This information can be used to devise special excitation geometries, e.g. for acoustic microscopes.

The interface between a copper layer on an aluminum substrate is studied and $V(\theta)$ curves are measured for artificially induced defects. Measurements indicate that the Lamb wave modes are more sensitive than the Rayleigh-like mode. Using the Lamb wave modes images of the interface are produced. The inhomogeneities at the interface are detected with a high sensitivity and with a diffraction limited resolution.

Improving the conical wave and optimizing its size for the Lamb wave modes will help produce better images because of better selectivity among various modes and higher signal to noise ratio.

\section{References}

[1] H.L. Bertoni and T. Tamir, "Unified theory of Rayleigh angle phenomena for acoustic beams at liquid-solid interfaces", Appl. Phys., vol.2, pp.157-172, 1973.

[2] H.L. Bertoni and T. Tamir, "Characteristics of wedge transducers for acoustic surface waves", IEEE Trans Sonics Ultrason., vol. 22, pp. 415-420, 1975 .

[3] H.L. Bertoni, "Coupling layers for efficient wedge transducers", IEEE Trans. Sonics Ultrason., vol. 22, pp. 421-430, 1975.

[4] J. Fraser, P.T. Khuri-Yakub and G.S. Kino, "The design of efficient broadband wedge transducers," Appl. Phys. Lett., vol. 32, pp. 698700,1978 .

[5] L.M. Brekovskikh, Waves in Layered Media, (Academic Press, New York, 1960).
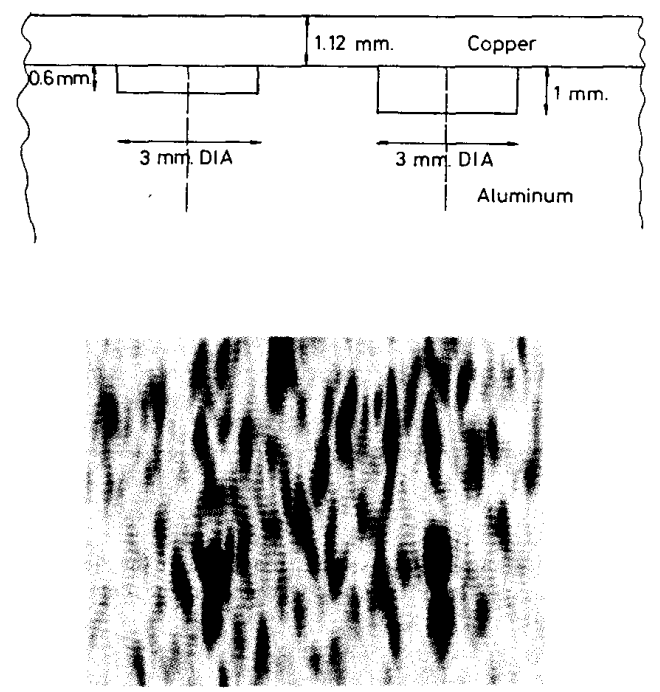

Figure 7: Lamb wave image of artificially induced flaws at the interface between a $1.12 \mathrm{~mm}$ thick copper layer and an aluminum substrate at $2 \mathrm{MHz}$.

[6] D.E. Chimenti, A.H. Nayfeh and D.L. Butler, "Leaky Rayleigh waves on a layered halfspace," J. Appl. Phys., vol. 53, pp. 170176,1982

[7] D.L. Folds and C.D. Loggins, "Transmission and reflection of ultrasonic waves in layered media," J. Acoust. Soc. Am., vol. 62, pp. 1102-1109, 1977.

[8] D.B. Bogy and S.M. Gracewski, "Reflection coefficient for plane waves in a fluid incident on a layered elastic half-space," $J$. Appl. Mech., vol. 50, pp. 405-414, 1983.

[9] G.W. Farnell and E.L. Adler, "Elastic Wave propagation in thin layers," in Physical Acoustics, edited by W.P. Mason and R.N. Thurston, (Academic, New York, 1972) vol. IX, pp. 35-127,

[10] A.H. Nayfeh, and D.E. Chimenti, "Reflection of finite acoustic beams from loaded and stiffened half-spaces," J. Acoust. Soc. Am. vol. 75 , pp. 1360-1368, 1984 .

[11] A. Atalar and H. Köymen, "Use of a conical axicon as a surface acoustic wave focusing device", IEEE Trans. Ultrason. Ferro. and Freq. Cont., vol. 34 , pp. 53-63, 1987.

[12] F.C. Cuozzo, E.L. Cambiaggio, J-P Damiano, and E. Rivier, "Influence of elastic properties on Rayleigh wave scattering by normal discontinuities," IEEE Trans. Sonics Ulirason., vol. 24, pp. 280289,1977

[13] H. Köymen, A. Atalar, T. Ciloğlu, M. Önder, Cetin Uzel and H. Yavuz, "Imaging flaws close to surface using focused surface acoustic waves" in Proc. IEEE Ulirasonics Symposium, pp. 755758,1986 .

[14] S. Ayter, "Focusing surface waves using conical transducers," in Proc. of 1987 IEEE Ultrason. Symp., pp. 301-304, 1987. 